\title{
Geomapping of Traumatic Spinal Cord Injury in Canada and Factors Related to Triage Pattern
}

\author{
Christiana L. Cheng,, Vanessa K. Noonan, Jayson Shurgold,, Jason Chen, Carly S. Rivers, \\ Hamid Khaleghi Hamedani, Suzanne Humphreys, Christopher S. Bailey, Najmedden Attabib, \\ Jean-Marc Mac Thiong, Michael Goytan, Jerome Paquet, Richard Fox, Henry Ahn, Brian K. Kwon, \\ Daryl R. Fourney ${ }^{10}$ and the RHSCIR Network ${ }^{1}$
}

\begin{abstract}
Current research indicates that more than half of patients with traumatic spinal cord injury (tSCI) experience delays in transfer and receive surgery $>24 \mathrm{~h}$ post-injury. The objectives of this study were to determine the geographic distribution of tSCI in Canada relative to specialized treatment facilities, to assess clinical and logistical factors at play for indirect admissions to those facilities, and to explore differences in current time to admission and simulated scenarios in an attempt to assess the potential impact of changes to triage protocols. This study included data from 876 patients with tSCI enrolled in the prospectively collected acute Rick Hansen Spinal Cord Injury Registry (RHSCIR) between January 1, 2010 and December 31, 2013 for whom there were data on the location of their injury. Patients transported directly to a RHSCIR acute facility were more likely to reach the facility within $1 \mathrm{~h}$ of injury, whereas those transported indirectly were more likely to arrive $7 \mathrm{~h}$ later. Considering the injuries occurring within $40 \mathrm{~km}$ of a RHSCIR acute facility $(n=323), 249$ patients $(77 \%)$ were directly and $74(23 \%)$ were indirectly admitted. In the multivariate regression analysis, only older age and longer road distance remained significantly associated with being indirectly admitted to a RHSCIR facility. Compared with the current status, the median time to admission decreased by $20 \%(3.5 \mathrm{~h})$ in the $100 \%$ direct admission scenario, and increased by $102 \%(8.9 \mathrm{~h})$ in the $100 \%$ indirect admission scenario.
\end{abstract}

Keywords: acute SCI; geographic information science; SCI center; trauma center; triage

\section{Introduction} MMEDIATELY FOLLOWING the onset of traumatic spinal cord injury (tSCI), patients are at significant risk of neurological deterioration resulting from secondary injury to the spinal cord. ${ }^{1}$ Prompt recognition of $\mathrm{tSCI}$, appropriate triage, and timely access to specialized care are critical. Evidence-based guidelines developed by expert consensus recommend early transport of patients sustaining an acute tSCI to a SCI-specialized center, ${ }^{2-6}$ and early surgical treatment within $24 \mathrm{~h}$ of injury ${ }^{7-9}$ to achieve optimal outcome. However, despite these guidelines and the growing evidence supporting the benefits of expeditious transfer to a SCI center, current research indicates that more than half of patients with tSCI experience delayed admission to SCI centers, ${ }^{10,11}$ and receive surgery $>24$ h post-injury. ${ }^{12}$

To understand the barriers to achieving optimal care delivery, recent efforts have aimed at delineating the processes of the clinical journey from point of injury to commencement of specialized care and at identifying potential correctible delays. ${ }^{10,12-14}$ Processes measured in these studies included pre-hospital time, time spent in an intermediate nonspecialized center, time from injury to specialized center, time to assessment by a spine surgeon, time to

\footnotetext{
${ }^{1}$ Rick Hansen Institute, Vancouver, British Columbia, Canada.

${ }^{2}$ Division of Orthopaedics, Department of Surgery, Western University, London, Ontario, Canada.

${ }^{3}$ Horizon Health Network, Dalhousie University, Saint John, New Brunswick, Canada.

${ }^{4}$ Department of Surgery, Faculty of Medicine, University of Montreal, Montreal, Quebec, Canada.

${ }^{5}$ Sections of Orthopedics and Neurosurgery, University of Manitoba, Winnipeg, Manitoba, Canada

${ }^{6}$ Sciences Neurologiques, Université Laval, Québec, Quebec, Canada.

${ }^{7}$ Faculty of Medicine and Dentistry, Division of Neurosurgery, University of Alberta, Edmonton, Alberta, Canada.

${ }^{8}$ University of Toronto Spine Program, Toronto, Ontario, Canada.

${ }^{9}$ Department of Orthopaedics, University of British Columbia, Vancouver, British Columbia, Canada.

${ }^{10}$ Division of Neurosurgery, University of Saskatchewan, Royal University Hospital, Saskatoon, Saskatchewan, Canada.
}

(C) Christiana L. Cheng et al., 2017; Published by Mary Ann Liebert, Inc. This Open Access article is distributed under the terms of the Creative Commons Attribution Noncommercial License (http://creativecommons.org/licenses/by-nc/4.0/) which permits any noncommercial use, distribution, and reproduction in any medium, provided the original author(s) and the source are credited 
decision regarding surgical management, and time to surgery. Results from these studies revealed that patients who were sent indirectly to the specialized center spent an average of 9-33 $\mathrm{h}$ in the intermediate center, ${ }^{11,12,15}$ and received surgery $14 \mathrm{~h}$ later than those who were admitted directly to the specialized center. ${ }^{11}$ These findings suggest that direct admission to the specialized center would accelerate access to appropriate care; however, this might not be possible in cases requiring immediate medical stabilization caused by injury severity or geographic limitations.

Given that injuries can occur in both urban and very remote areas, there is a need to ensure that triage procedures account for differences in care depending on the geographic location of injury. In urban settings where SCI-specialized centers are accessible within a short time frame and, therefore, direct admission is feasible, the degree to which direct admission is occurring has not been examined for $\mathrm{tSCI}$. There are indications from the general trauma literature that despite the published guidelines, patients are still being triaged to a non-trauma center even when they are injured within vicinity of the trauma center. A study in California reported that $42 \%$ of all indirect admissions were injured within 10 miles $(16.1 \mathrm{~km})$ of the trauma center ${ }^{16}{ }^{16}$ whereas another study in Toronto reported $63 \%$ of indirect admissions were injured within 5 miles $(8 \mathrm{~km})$ of the trauma center, ${ }^{17}$ in both cases patients could have bypassed nonspecialized emergency rooms in favor of being delivered directly to a trauma center as specified by evidence-based field trauma triage protocols. ${ }^{18}$

To identify opportunities to improve timely access to SCIspecialized care in Canada, we must first understand the current status of tSCI geography and triage patterns across the country. The purpose of this study was to describe the geographic distribution of tSCI and calculate the road distances between injury and the SCI center. Further, we determined the status of indirect admission to a SCI center and its associated factors for the cohort of injuries occurring in close proximity to a SCI center that could potentially be directly transported. Finally, to provide some context for the impact of a potential change in triage protocol, the Access to Care and Timing (ACT) simulation model for tSCI care ${ }^{19,20}$ was applied to explore differences in time to admission between current status and simulated scenarios. This study aimed to assess the basis of triage patterns in Canadian urban settings and compare care to the recommended guidelines.

\section{Methods}

\section{Study design}

This retrospective study used data from the Rick Hansen Spinal Cord Injury Registry (RHSCIR), which is a prospective registry of patients with a new tSCI from 18 SCI acute and 13 rehabilitation facilities located in 16 cities across Canada. All RHSCIR participating acute facilities were Level I/II trauma centers at the time of study, ${ }^{21,22}$ and all RHSCIR facilities would be considered to be acute SCI specialty centers as defined by the recommended attributes in Parent and coworkers and Noonan and coworkers. ${ }^{4,22}$ Each participating facility obtained institutional research ethics board approval to enroll patients. A core data set was collected for all registrants, whereas an expanded data set was collected for those who provided informed consent. Further details about RHSCIR have been previously published. ${ }^{23,24}$

This study included patients who were admitted to an acute RHSCIR facility between January 1, 2010 and December 31, 2013, provided informed consent for the collection of the expanded data set, and had level of injury at L2 and above (Fig. 1). The fairly narrow time range was chosen to minimize the effect of evolving care practice, ${ }^{2,25}$ specifically as a result of the guidelines regarding early transport to SCI center that have been published in recent years. ${ }^{2-5}$

\section{Study variables}

Patient variables included age at time of injury and gender. Injury variables included injury mechanism (fall, transport, sports, assault, other), kinetic energy related to injury mechanism (low, high energy [i.e., any injury involving a high velocity situation, e.g., gunshot wound, pedestrian struck, auto crash $>65 \mathrm{~km} / \mathrm{h}$ or falls $>6 \mathrm{~m}]$ ), Glasgow Coma Scale (GCS) at admission (severe to

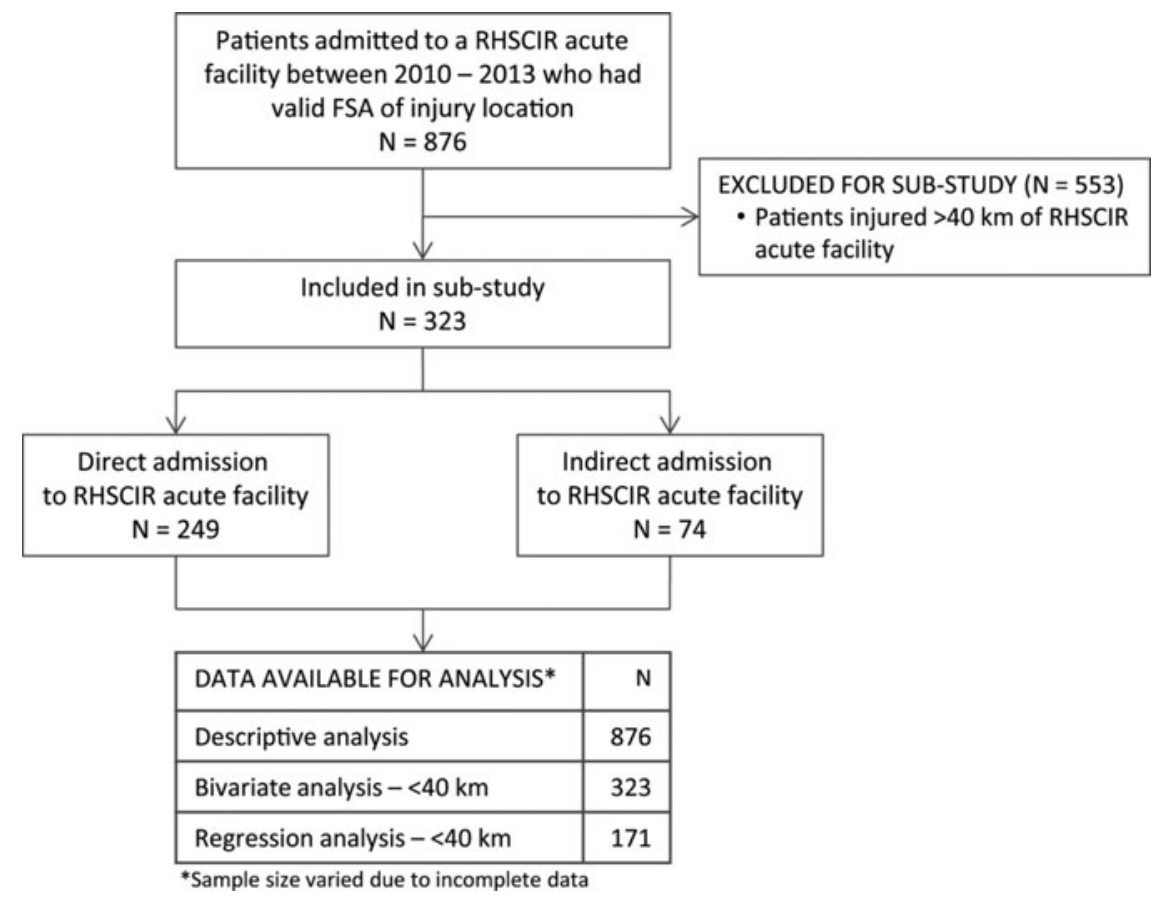

FIG. 1. Flow of patients in the RHSCIR and selection of patients for analysis. RHSCIR; Rick Hansen Spinal Cord Injury Registry; FSA, Forward Sortation Area. 
moderate decreased level of consciousness, 3-12; mildly altered level of consciousness, 13-15), Injury Severity Score (ISS) at admission $(\leq 25 ;>25$ indicating major trauma to another body region in addition to the spine), ${ }^{26}$ neurological classification by American Spinal Injury Association (ASIA) Impairment Scale (AIS) (A, B, C, D) and neurological level at admission (high cervical, C1-C4; low cervical, C5-T1; thoracic, T2-T10; thoracolumbar, T11-L2), both of which were derived from the International Standards for Neurological Classification of SCI (ISNCSCI) examination. ${ }^{27}$ Missing ISS data were imputed based on the joint distribution of AIS and neurological level at admission.

Geographical factors. The RHSCIR acute facility where patient was admitted and the forward sortation area (FSA) of injury location were obtained from RHSCIR. FSA is a well-defined zone within a larger geographic region based on the first three characters of the postal code. ${ }^{28}$

System factors. The following durations (in hours) were calculated: time between injury and admission to non-RHSCIR acute facility, and time between injury and admission to RHSCIR acute facility.

Outcome variable. The primary outcome variable in this study was triage pattern (direct vs. indirect admission). Direct admission was defined as occurring when patients were transported directly from site of injury to a RHSCIR acute facility; indirect admission was defined as arrival at a RHSCIR acute facility from site of injury via one or more hospitals.

\section{Geomapping analysis}

MapQuest application program interface (API) (MapQuest Inc.) integrated with SAS software was applied to determine the centroid of each FSA, yielding a unique combination of latitude and longitude coordinates of each FSA's geometric center. These coordinates and that of RHSCIR acute facilities, as calculated by Geographic Information Systems (GIS) technology, were used to determine the road distance between injury and RHSCIR facility. Road distance was categorized as either $<40 \mathrm{~km}, 40-350 \mathrm{~km}$, or $>350 \mathrm{~km}$. The lower category $(40 \mathrm{~km})$ was based on trauma destination decision guidelines that advise trauma patients be transported to the most appropriate trauma center if transport time would be $<40 \mathrm{~min} ;{ }^{29}$ equivalent to $\sim 40 \mathrm{~km}$ if driving at $60 \mathrm{~km} / \mathrm{h}$. The upper category $(350 \mathrm{~km})$ was based on the average distance traveled by helicopter with one tank of fuel if one was deployed for transportation of patients.

The geographical distribution of tSCI was estimated and illustrated using Maptitude 2013 (Caliper Corporation) overlaid with population density obtained from Demography Division, Statistics Canada (2011). ${ }^{30}$ Positions were marked for each patient randomly within the reported FSA of injury.

\section{Statistical analysis}

Descriptive analysis was performed to describe the entire study population. Further analysis was performed with the cohort of individuals whose injury location was within $40 \mathrm{~km}$ of the RHSCIR acute facility, within which the direct admissions were compared with the indirect admissions using bivariate analysis. Differences among medians of continuous variables were compared using Wilcoxon rank sum test, and differences among percentages of categorical variables were examined using the $\mathrm{X}^{2}$ test. A multivariate logistical regression model was developed to examine the variables associated with indirect admission. Variables that were statistically and clinically significant, and provinces with indirect admissions within $40 \mathrm{~km}$ of a RHSCIR acute facility, were considered for inclusion in the multivariate regression model.
A sensitivity analysis was performed to examine cohort selection bias between patients included and those who were excluded because of missing FSA data. Cohorts were compared based on age, gender, injury mechanism, AIS, and neurological level at admission.

A $p$ value of $<0.05$ was considered to be statistically significant. All analyses were performed using SAS software, Version 9.4 of the SAS System for Windows, copyright (c) 2013, SAS Institute Inc., Cary, NC.

\section{Simulation modeling}

Age and gender distribution, probability of triage pattern (direct/ indirect admission), and distribution of road distance between injury location and RHSCIR acute facility, all of which were derived from the analysis cohort in this study, were used in the ACT model to explore the impact of triage pattern on process of care.

Three scenarios were analyzed: 1) current direct admission rate (baseline), 2) 100\% direct admission, and 3) $100 \%$ indirect admission. The outcome of interest was time to admission, which was defined as the duration between time of injury and admission to a RHSCIR acute facility. The model was run 15 times and the final outcome was the average of the 15 replications. Outcome was calculated with the following equation: outcome $=($ time to admission $_{\text {scenario }}$ - time to admission baseline/time to admission baseline.

\section{Results}

Of the 876 patients who had FSA data on injury location (Fig. 1), $77 \%$ were male and the median age was 49 years. The top two injury mechanisms were fall $(49 \%)$ and transport (28\%). Other injury characteristics are described in Table 1 . Median road distance between site of injury and RHSCIR acute facility was $91 \mathrm{~km}$ (interquartile range [IQR] 19.6 and 223.5). Admission to RHSCIR facility for all patients (directly and indirectly) took a median of $5 \mathrm{~h}$. For the indirect admissions, median time to the first non-RHSCIR acute facility was $1.3 \mathrm{~h}$.

A sensitivity analysis revealed that patients who were excluded from the analysis because of missing FSA data $(n=509)$ were significantly younger (median age 44 vs. $49, p<0.0001$ ), more likely to be male $(83 \%$ vs. $77 \%, p=0.01)$, more likely to have a sports-related injury $(17 \%$ vs. $12 \%, p=0.007)$ or a transport-related injury ( $42 \%$ vs. $28 \%, p<0.0001$ ), than those included in the analysis. Injury severity as measured by AIS and neurological level at admission was not significantly different between the two groups.

\section{Geographic distribution of $t S C l$ in Canada}

Figure 2 describes the geographic distribution of tSCI in each province for patients admitted to a RHSCIR acute facility. A dispersed pattern is observed in provinces with geographically dispersed populations, such as the western/central provinces (British Columbia, Alberta, Saskatchewan), and a confined pattern is observed for provinces where the population density concentrates in urban cities, such as Ontario and Quebec. Figure 3 describes the geographical distribution of injuries by distance range $(<40 \mathrm{~km}$, $40-350 \mathrm{~km},>350 \mathrm{~km}$ ) for each Canadian province. Newfoundland had the largest proportion of injuries occurring $>350 \mathrm{~km}$ from the RHSCIR acute facility (58\%). In the $40-350 \mathrm{~km}$ range, New Brunswick had the largest proportion of injuries (80\%) followed by Saskatchewan, Quebec, and Nova Scotia (63\%, 55\%, and 59\%, respectively). Manitoba and Ontario had the most injuries in the $<40 \mathrm{~km}$ range (50\% and 49\%, respectively). British Columbia had similar proportions distributed across the three distance ranges. 
Table 1. Patient Characteristics of InJuries Occurring within 40 KM of a RHSCIR Acute Facility, and Direct Admission versus Indirect Admission to the RHSCIR Acute Facility

\begin{tabular}{|c|c|c|c|c|c|}
\hline \multirow[b]{2}{*}{ Variable } & \multirow[b]{2}{*}{ Total $(\mathrm{n}=876)$} & \multirow[b]{2}{*}{$\begin{array}{l}\text { Injured }<40 \mathrm{~km} \\
\quad(\mathrm{n}=323)\end{array}$} & \multicolumn{3}{|c|}{ Injured $<40 \mathrm{~km}$} \\
\hline & & & $\begin{array}{l}\text { Direct admission } \\
\quad(\mathrm{n}=249)\end{array}$ & $\begin{array}{l}\text { Indirect admission } \\
\qquad(\mathrm{n}=74)\end{array}$ & $\mathrm{p}$ value \\
\hline Age at injury; median (IQR) & $49(31,64)$ & $53(37,65)$ & $49(34,64)$ & $61.5(48,69)$ & $<0.0001$ \\
\hline Male gender; $n(\%)$ & $675(77.1)$ & $252(78)$ & $195(78.3)$ & $57(77.0)$ & 0.8145 \\
\hline Injury mechanism; $n(\%)$ & & & & & 0.3828 \\
\hline Fall & $431(49.2)$ & $198(61.3)$ & $147(59.0)$ & $51(68.9)$ & \\
\hline Transport & $245(28.0)$ & $58(18.0)$ & $50(20.1)$ & $8(10.8)$ & \\
\hline Sports & $104(11.9)$ & $27(8.4)$ & $22(8.8)$ & $5(6.8)$ & \\
\hline Assault & $38(4.3)$ & $22(6.8)$ & $17(6.8)$ & $5(6.8)$ & \\
\hline Other & $58(6.6)$ & $18(5.6)$ & $13(5.2)$ & $5(6.8)$ & \\
\hline Energy of injury; $n(\%)$ & & & & & 0.0034 \\
\hline High & $379(45.3)$ & $113(36.3)$ & $97(40.8)$ & $16(21.9)$ & \\
\hline Low & $457(54.7)$ & $198(63.7)$ & $141(59.2)$ & $57(78.1)$ & \\
\hline Glasgow Coma Scale; $n(\%)$ & & & & & 0.34 \\
\hline $3-12$ & $78(10.4)$ & $26(9.7)$ & $22(10.8)$ & - & \\
\hline $13-15$ & $669(89.6)$ & $243(90.3)$ & $181(89.2)$ & $62(93.9)$ & \\
\hline Injury Severity Score; $n(\%)$ & & & & & 0.3189 \\
\hline$\leq 25$ & $440(57.9)$ & $173(62.5)$ & $129(60.9)$ & $44(67.7)$ & \\
\hline$>25$ & $320(42.1)$ & $104(37.5)$ & $83(39.2)$ & $21(32.3)$ & \\
\hline AIS at admission; $n(\%)$ & & & & & 0.3003 \\
\hline AIS A & $260(35.0)$ & $86(30.9)$ & $70(33.7)$ & $16(22.9)$ & \\
\hline AIS B & $68(9.1)$ & $24(8.6)$ & $19(9.1)$ & $5(7.1)$ & \\
\hline AIS C & $151(20.3)$ & $53(19.1)$ & $38(18.3)$ & $15(21.4)$ & \\
\hline AIS D & $265(35.6)$ & $115(41.4)$ & $81(38.9)$ & $34(48.6)$ & \\
\hline Neurological level at admission; $n(\%)$ & & & & & 0.1038 \\
\hline High cervical (C1-C4) & $189(27.6)$ & $74(28.2)$ & $50(25.9)$ & $24(38.1)$ & \\
\hline Low cervical (C5-T1) & $230(33.6)$ & $94(35.9)$ & $77(39.9)$ & $17(27.0)$ & \\
\hline Thoracic (T2-T10) & $119(17.4)$ & $38(14.5)$ & $31(16.1)$ & $7(11.1)$ & \\
\hline Thoracolumbar (T11-L2) & $147(21.5)$ & $50(19.1)$ & $35(18.1)$ & $15(23.8)$ & \\
\hline $\begin{array}{l}\text { Time to non-RHSCIR acute facility (h); } \\
\text { median (IQR) }\end{array}$ & $1.3(0.8,3.3)$ & $1.1(0.6,5.2)$ & N/A & $1.1(0.6,5.2)$ & N/A \\
\hline $\begin{array}{l}\text { Time to RHSCIR acute facility (h); } \\
\text { median (IQR) }\end{array}$ & $5.1(1.7,11.8)$ & $1.5(0.8,6.9)$ & $1.1(0.7,2.9)$ & $7.9(4.3,40)$ & $<0.0001$ \\
\hline Road distance $(\mathrm{km})$; median (IQR) & $91.1(19.6,223.5)$ & $13.1(6.1,24)$ & $10.7(5.6,19.6)$ & $21.3(10.3,32.4)$ & $<0.0001$ \\
\hline
\end{tabular}

Data within cell $<5$ not shown.

Bold $\mathrm{p}$ values indicate statistical significance.

IQR, interquartile range; AIS, American Spinal Injury Association (ASIA) Impairment Scale; RHSCIR, Rick Hansen Spinal Cord Injury Registry.

\section{Triage pattern from $\mathrm{tSCl}$ to $\mathrm{SCl}$ center within $40 \mathrm{~km}$}

Considering the injuries occurring within $40 \mathrm{~km}$ of a RHSCIR acute facility $(n=323), 249$ patients $(77 \%)$ were directly admitted and $74(23 \%)$ were indirectly admitted (Table 1$)$. The percentage of direct admission for injuries within $40 \mathrm{~km}$ for each province varied across Canada ranging from $53 \%$ to $100 \%$ (Fig. 4). The triage pattern (direct vs. indirect admission) in five major cities is illustrated in Figure 5. Four cities demonstrated a lack of geographic distinction between direct and indirect admissions, whereas Quebec City had $100 \%$ direct admissions regardless of distance (Fig. 5e). A histogram of admission frequency (direct vs. indirect) by road distance between injury location and RHSCIR facility reveals a rightly-skewed distribution for the direct admission, suggesting that more direct admissions were observed for shorter road distances, whereas no clear pattern was observed for the indirect admissions (Fig. 6).

To explore the factors associated with patients being triaged indirectly to the RHSCIR facility when they were within the vicinity $(<40 \mathrm{~km})$, this group was compared with those directly ad- mitted. The bivariate analyses showed that the indirect admission group was significantly older (62 vs. $49, p<0.0001$ ), more likely to have had a low-energy injury ( $78 \%$ vs. $59 \%, p=0.003$ ), more likely to have been injured relatively farther away from the RHSCIR facility $(21.3 \mathrm{~km}$ vs. $10.7 \mathrm{~km}, p<0.0001)$, and more likely to have taken longer to reach the RHSCIR facility ( $8 \mathrm{~h}$ vs. $1 \mathrm{~h}, p<0.0001$ ) than the directly admitted group (Table 1). However, the median time to the first hospital (non-RHSCIR) was $1 \mathrm{~h}$ for the indirectly admitted group.

In the multivariate regression analysis, only older age and longer road distance remained significantly associated with being indirectly admitted to a RHSCIR facility, adjusted for energy of injury, ISS, and neurological classification (Table 2). Specifically, the risk of not being directly admitted increased by $2.7 \%$ for every increased year of age and increased by $5.3 \%$ for every increased kilometer of road distance between the injury location and the RHSCIR facility. None of the injury factors, including energy of injury, injury severity, and extent of neurological deficit were associated with triage pattern for injuries occurring within $40 \mathrm{~km}$ of a RHSCIR acute facility. 


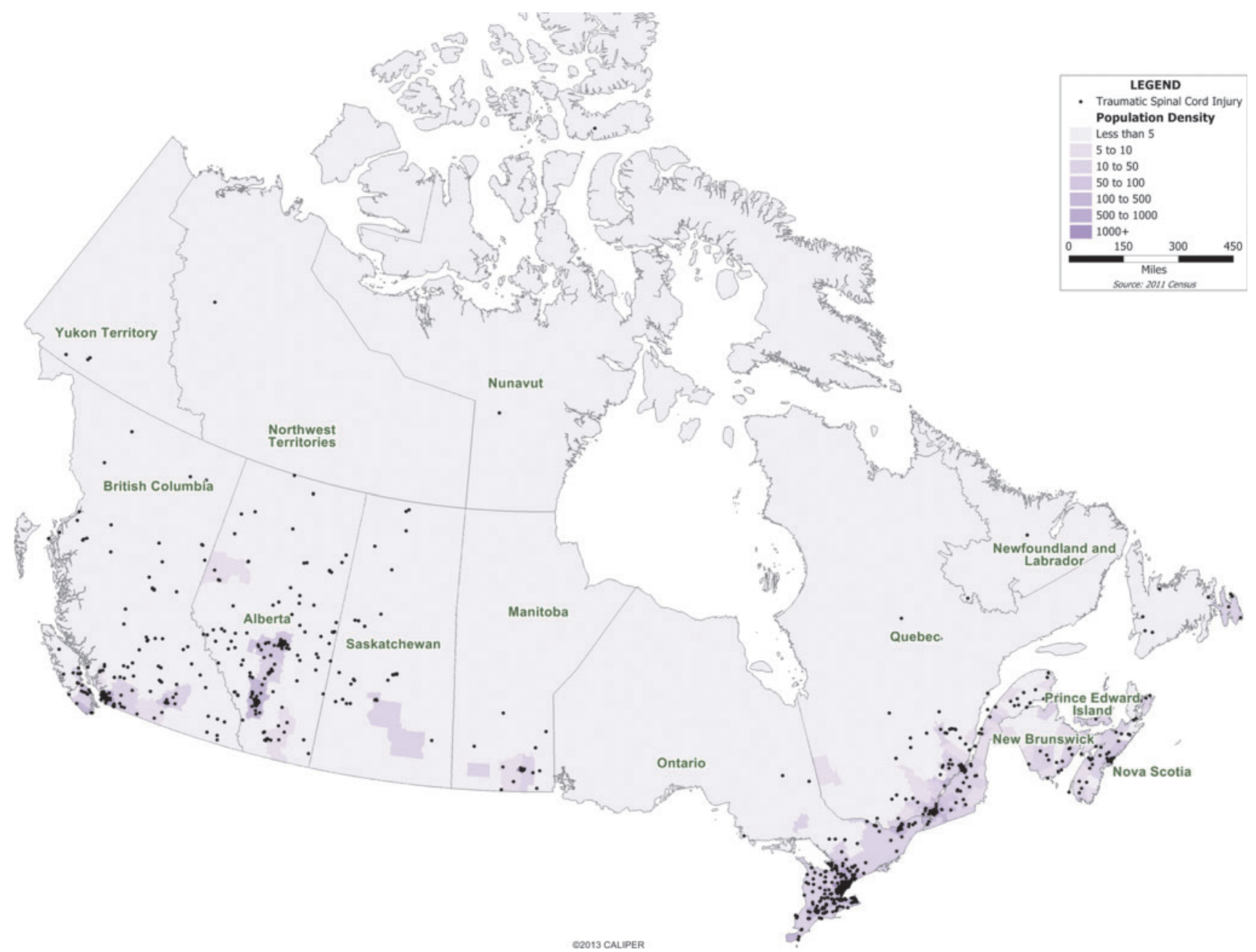

FIG. 2. Geomapping of traumatic spinal cord injury admitted to Rick Hansen Spinal Cord Injury Registry (RHSCIR) acute facilities in 2010-2013.

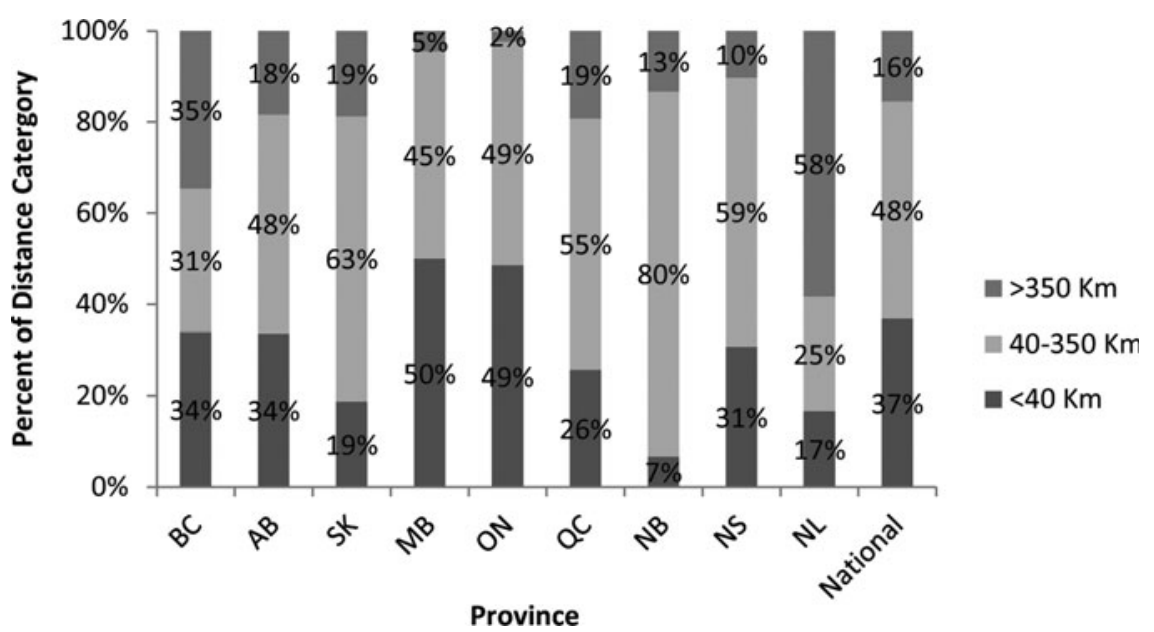

FIG. 3. Proportion of traumatic spinal cord injuries that occurred within $40 \mathrm{~km}, 40-350 \mathrm{~km}$, and beyond $350 \mathrm{~km}$ of a Rick Hansen Spinal Cord Injury Registry (RHSCIR) acute facility by province. BC, British Columbia; AB, Alberta; SK, Saskatchewan; MB, Manitoba; ON, Ontario; QC, Quebec; NB, New Brunswick; NS, Nova Scotia; NL, Newfoundland. 


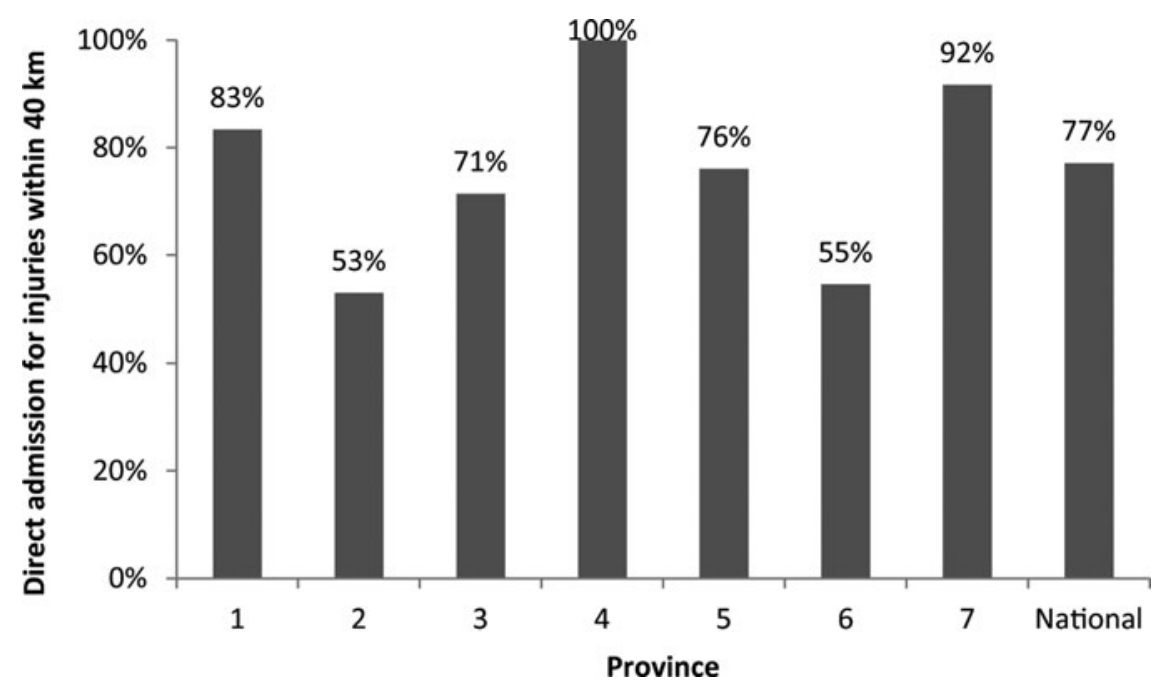

FIG. 4. Direct admission of patients injured within $40 \mathrm{~km}$ of a Rick Hansen Spinal Cord Injury Registry (RHSCIR) acute facility by province. Each province was randomly assigned a number. Provinces with a sample size $<5$ were not included.

\section{Simulation modeling}

The impact of triage pattern on process of care was explored with simulation modeling. Compared with the current status, the median time to admission decreased by $20 \%(3.5 \mathrm{~h})$ in the $100 \%$ direct admission scenario; and increased by $102 \%(8.9 \mathrm{~h})$ in the $100 \%$ indirect admission scenario.

\section{Discussion}

This study of the geomapping of tSCI in Canada revealed a wide spatial distribution and a varying pattern of injuries across provinces. Nearly $50 \%$ of tSCI occurred at a distance range of 40$350 \mathrm{~km}$ of a RHSCIR acute facility, which is a SCI center that is part of a Level I/II trauma center. More than one third of tSCI occurred within $40 \mathrm{~km}$ and, despite the close proximity, $23 \%$ of patients were not taken directly to the RHSCIR acute facility. Patients transported directly to a RHSCIR acute facility were more likely to reach the facility within $1 \mathrm{~h}$ of injury, whereas those transported indirectly were more likely to arrive a median of $7 \mathrm{~h}$ later. We observed that indirect admission for injuries within $40 \mathrm{~km}$ was associated with two independent factors: older age and greater distance between injury location and the RHSCIR acute facility.

\section{Relationship to previous literature}

The $40 \mathrm{~km}$ cutoff was selected because it is the maximum distance patients should be transported directly to definitive care, bypassing closer non-trauma centers, if these patients meet trauma criteria ${ }^{29,31}$ Within this range, distance to the RHSCIR facility should have minimal influence on triage decision. However, our study found that this does not necessarily occur, and is supportive of another Canadian study of general traumatic injuries reporting $47 \%$ of indirect admission for injuries located within $30 \mathrm{~min}$ of an Ontario trauma center, and that the added distance, as short as 1 mile $(1.6 \mathrm{~km})$, between the closest non-trauma hospital and the trauma center decreases the likelihood that the patient would be triaged to a trauma center. ${ }^{17}$

In cases in which the closest hospital is a nonspecialized trauma center, admission to the specialized center might be further delayed, as patients would be expected to be triaged to the closer nonspecialized trauma center according to the Golden Hour concept. ${ }^{32}$ This could be an issue in many cities where multiple trauma centers have been established to serve the dense population, ${ }^{21}$ but not all centers have SCI specialty. Given the lack of designated SCI centers in most provinces, currently many provincial triage destination decision guidelines consider any trauma center with orthopedic and neurosurgical capability as being appropriate for treating patients with suspected tSCI. For example, in British Columbia, two such additional trauma centers are located within $40 \mathrm{~km}$ of the acute SCI center (the RHSCIR acute facility). Based on this guideline, 12 trauma centers in Ontario are considered capable of providing "definitive care" for SCI, ${ }^{12} 7$ of which are RHSCIR acute facilities. Although we did not differentiate indirect admission coming from a non-trauma center or trauma center, unpublished data from the RHSCIR show that most of the indirect admissions were from trauma centers, indicating an awareness within the trauma community of RHSCIR facilities having a SCI specialty. In contrast to the rest of Canada, the Quebec provincial government has designated two trauma centers (the RHSCIR acute facilities in Quebec City and in Montreal) as centers of expertise in SCI; this designation might play a key role in achieving the $100 \%$ direct admission for Quebec City (Fig. 5e). Designating centers as having expertise in SCI would also position the center to forge formal collaboration with other hospitals treating seriously injured patients to ensure standards of care.

The accessibility of multiple trauma centers with orthopedic/ neurosurgical capability might contribute to the lack of association between neurological deficit and direct admission to a RHSCIR facility. This is consistent with previous literature that revealed that recognition of tSCI at the scene does not increase the likelihood of direct transport to a SCI center. ${ }^{10}$ However, challenges with recognizing tSCI at the scene might be the underlying reason for not triaging to the center. Given the heterogeneity of tSCI, the clinical symptoms may be unrecognizable. Concurrent injuries such as traumatic brain injury (TBI) or decreased cognitive level may make it difficult to diagnose the tSCI, whereas low-energy injuries may not raise suspicion of a tSCI, ${ }^{10}$ and detection of fractures in older people could also be problematic. It would be of great value to document the tSCI missed at the scene to clarify how changes should be made to facilitate direct transfer of the missed population. Conversely, 

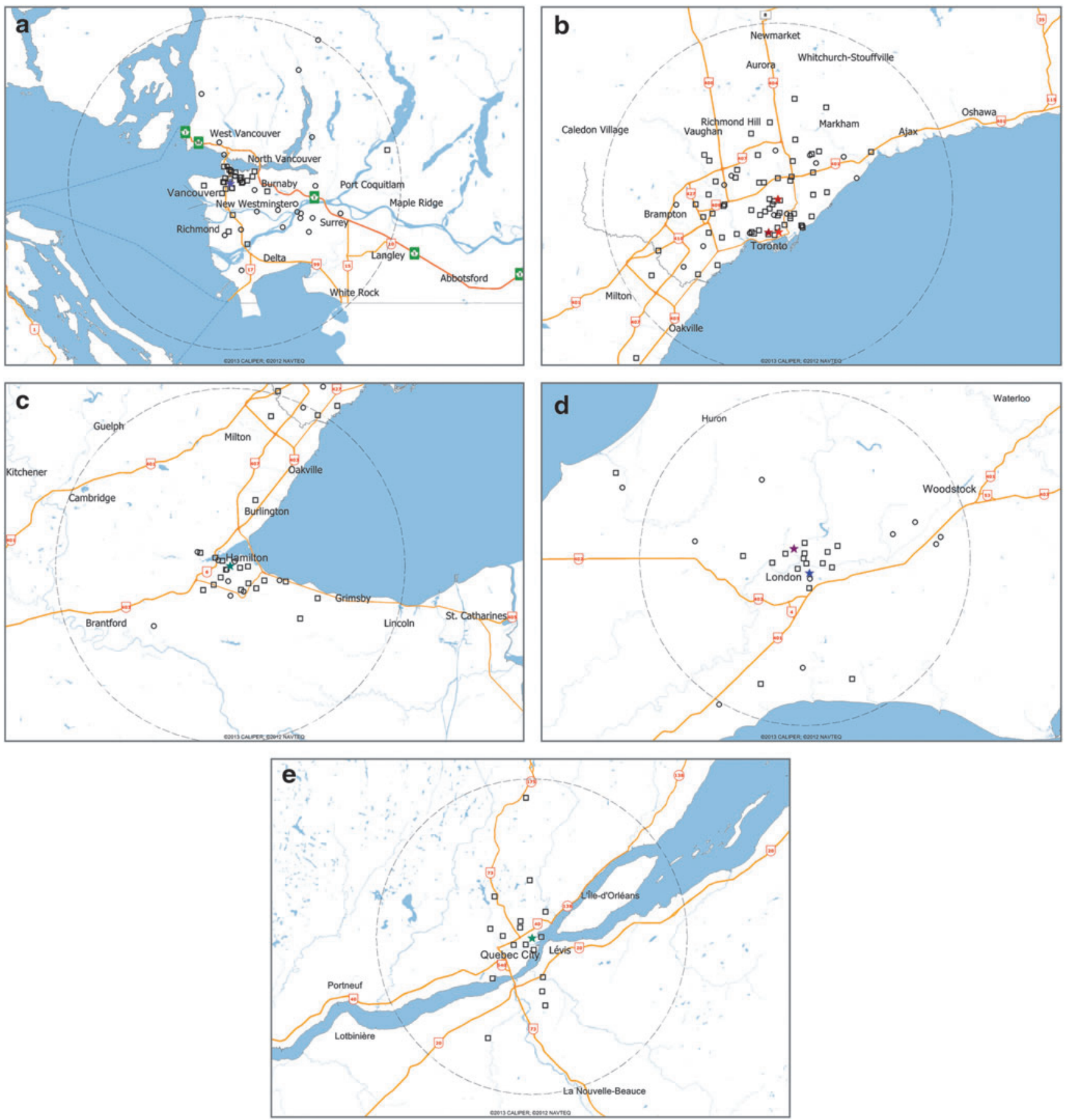

FIG. 5. Triage pattern of traumatic spinal cord injury to Rick Hansen Spinal Cord Injury Registry (RHSCIR) acute facility (direct vs. indirect) by city. (a) Vancouver, British Columbia; (b) Toronto, Ontario; (c) Hamilton, Ontario; (d) London, Ontario; (e) Quebec City, Quebec. Direct admission is represented by an open square; indirect admission is represented by an open circle. Location of the RHSCIR acute facility is represented by a star. Radius of $40 \mathrm{~km}$ from RHSCIR acute facility is approximated.

challenges in recognizing TBI during assessment of a suspected SCI have been reported, ${ }^{33}$ which might contribute to poor documentation and result in limited reporting of TBI such as we experienced with our data. Diagnosis of combined TBI and SCI is critical in defining an appropriate clinical pathway and rehabilitation treatment; therefore, future investigation into this issue is required.

Generally, patients with fall-related injuries experience delays in accessing a trauma center, whether the fall resulted in tSCI, TBI, or general trauma. ${ }^{12,17,34}$ Similar studies have found that the elderly are more likely to be transported to non-trauma center and have the greatest risk of not reaching a trauma center at all. ${ }^{10,12,17,34-37}$ Our finding of older age being associated with indirect admission to a RHSCIR acute facility is consistent with these studies, and suggests that an etiology other tha SCI was considered for this population in which cognitive deficits and mild neurological symptoms resulting from low-energy falls are common. This growing evidence highlights the need to explore the impact of delayed access to specialized care in the elderly population in future studies. ${ }^{38}$ 


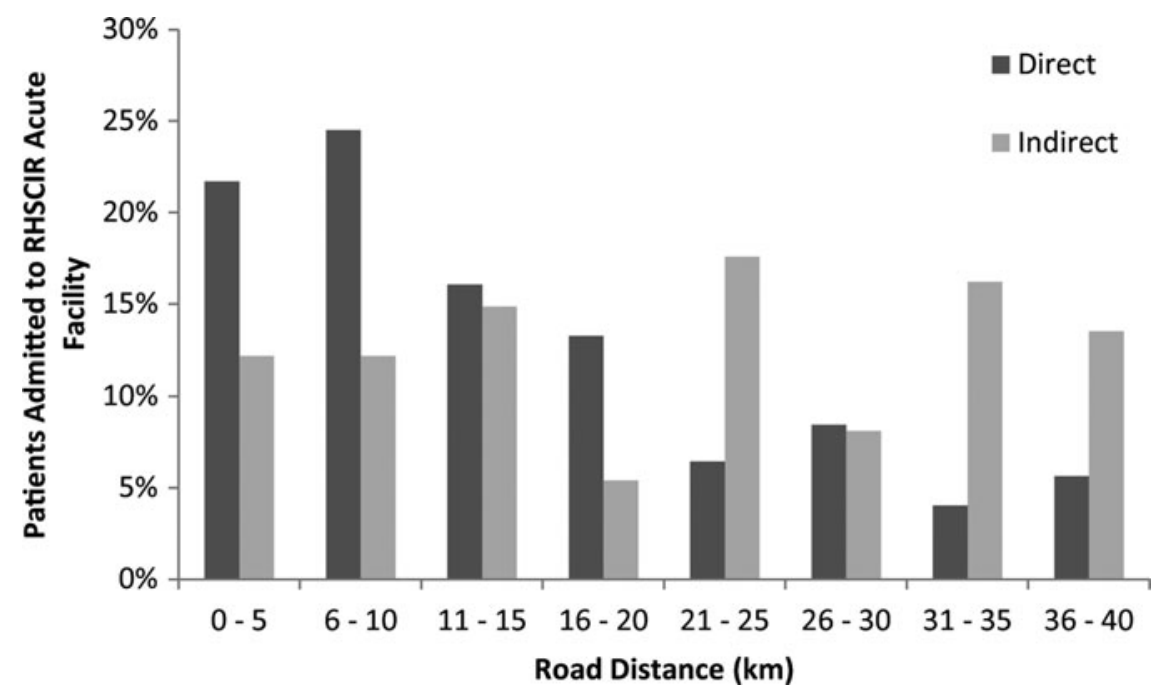

FIG. 6. Histogram of road distance between point of injury and Rick Hansen Spinal Cord Injury Registry (RHSCIR) acute facility. Directly admitted patients, dark gray bars; indirectly admitted patients, light gray bars.

Severely injured patients are associated with delayed admission to specialized or definitive trauma care ${ }^{10,16,39}$ because of the immediate medical attention required and provided at a closer hospital location. Considering the injury location in relation to the nearby trauma center, in addition to the acute SCI speciality center, would help to elucidate the impact of the injury severity on triage patterns. However, ISS as an index to measure severity of injury might not be sensitive enough to pick up the seriousness of the trauma, especially when considering specific injuries ${ }^{26}$ such as tSCI or burns. ${ }^{40}$ Further, ISS has not been validated for the tSCI population, and work is ongoing to develop an index for tSCI. ${ }^{41,42}$

\section{Implications}

This study shows that the majority of patients with tSCI in $\mathrm{Ca}$ nada are receiving the recommended care of early transport to a SCI center. For those injured with $40 \mathrm{~km}, 77 \%$ are being directly admitted to the RHSCIR acute facility with a median time of $1 \mathrm{~h}$, whereas the rest arrive indirectly with a median time of $8 \mathrm{~h}$ postinjury. However, we noted a highly variable interfacility transport time (IQR of 4.3-40 h). Similar delays associated with an indirect admission have been reported in other tSCI studies in which the median wait time is $8-12 \mathrm{~h},{ }^{10,12,14}$ and can be as long as $33 \mathrm{~h}^{15}$ Indirect admission could risk putting patients outside of the recommended $24 \mathrm{~h}$ post-injury time window to surgery, ${ }^{9,24}$ especially older patients who have a significantly longer wait time from admission to surgery than younger patients (37 vs. $19 \mathrm{~h}){ }^{38}$

One should address the question of whether enforcing direct admission to a SCI center would be the answer, and in what way this would impact the healthcare system. To explore this question, we used simulation modeling to analyze two opposing scenarios: $100 \%$ direct admission, which is a small increase (23\%) from the current status, and $100 \%$ indirect admission, which is a conceivable scenario given that older patients have been shown to experience more indirect admission, and that this population is growing. The scenario of $100 \%$ direct admission might appear to

Table 2. Multivariate Logistical Regression Analysis Predicting Variables Associated with Indirect Admission to a RHSCIR Acute Facility for Traumatic Spinal Cord Injury within 40 Km of the FaCility

\begin{tabular}{|c|c|c|c|c|c|}
\hline Variable & Estimates & Std. error & Odds ratio & $95 \% C I$ & $\mathrm{p}$ value \\
\hline Intercept & -2.42 & 0.92 & - & - & 0.0083 \\
\hline Age at injury & 0.03 & 0.01 & 1.03 & $1.00-1.05$ & 0.0255 \\
\hline \multicolumn{6}{|l|}{ Energy of injury } \\
\hline Low (reference) & - & - & - & - & - \\
\hline High & -0.42 & 0.47 & 0.66 & $0.26-1.66$ & 0.373 \\
\hline \multicolumn{6}{|l|}{ Injury Severity Score } \\
\hline$\leq 25$ (reference) & - & & - & - & - \\
\hline$>25$ & -0.07 & 0.55 & 0.93 & $0.32-2.74$ & 0.8965 \\
\hline \multicolumn{6}{|l|}{ Neurological category } \\
\hline C1-C4 AIS A & -0.11 & 0.77 & 0.90 & $0.20-4.07$ & 0.8865 \\
\hline C5-T1 AIS A & -0.72 & 0.93 & 0.49 & $0.08-3.04$ & 0.441 \\
\hline T2-L2 AIS A & -1.69 & 1.00 & 0.18 & $0.03-1.31$ & 0.0911 \\
\hline C1-T1 AIS B, C, D & -0.40 & 0.54 & 0.67 & $0.23-1.92$ & 0.4542 \\
\hline T2-L2 AIS B, C, D (reference) & - & & - & - & \\
\hline Road distance (km) & 0.05 & 0.02 & 1.05 & $1.02-1.09$ & 0.0007 \\
\hline
\end{tabular}

Bold $\mathrm{p}$ values indicate statistical significance.

CI, confidence interval; AIS, American Spinal Injury Association (ASIA) Impairment Scale; RHSCIR, Rick Hansen Spinal Cord Injury Registry. 
have little impact on the system, with savings of only $2 \mathrm{~h}$; however, the clinical significance of this cannot be underestimated, as early complications of SCI such as hypotension requiring vasopressors and respiratory distress requiring intubation need to be managed in the first few hours of injury to avoid further morbidity and mortality. ${ }^{43}$ Further, a recent study examining functional recovery at 1 year showed that very early treatment $(<8 \mathrm{~h})$ is beneficial for cervical trauma. ${ }^{8}$ The opposing scenario of $100 \%$ indirect admission resulted in doubling the time to reach a RHSCIR acute facility, implying that if the current triage practice is not rectified, more unnecessary indirect admissions and significant delays to specialized care could occur.

Currently, evidence supporting benefits of direct admission is limited, ${ }^{44}$ as most SCI studies examined the benefits of early admission instead (i.e., within $24 \mathrm{~h}$ post-injury), and none stratified the injury by distance to SCI center. ${ }^{2,4,5,10}$ However, direct admission for injuries within $40 \mathrm{~km}$ of a SCI center, and hence early access to care, offers many opportunities that would position patients and the healthcare system to gain substantially in the long term. Direct admission to a SCI center minimizes risk of exacerbating the injury that could arise during transfer and prolonged spinal immobilization. It also reduces variability in care, as practice and expertise differ in non-SCI centers. Patients who are directly admitted can participate in clinical trials testing promising neuroprotective and neuroregenerative treatments that usually have an effective time window of $<12 \mathrm{~h}^{45}$ From the health system perspective, reducing the unnecessary admission to the intermediate hospital and subsequent transportation to the RHSCIR acute facility can free up resources and save costs.

\section{Next steps/gaps}

To elucidate the benefits of direct admissions to specialized centers, comparing the outcomes between direct and indirect admission, as well as between those treated in RHSCIR facilities and those treated in non-RHSCIR facilities requires further investigation. It is also valuable to delineate which patient population (the specific type of SCI or age) would potentially benefit most from direct admission. Emerging evidence suggest that individuals with cervical injuries ${ }^{11}$ or with incomplete injuries ${ }^{24}$ have greater motor recovery if they have surgery within $24 \mathrm{~h}$ of injury. This could be especially relevant for the elderly, who are likely to be delayed in reaching a RHSCIR facility and being operated on; ${ }^{38}$ however, given the increased rates of pre-existing comorbidities ${ }^{46}$ and the higher likelihood of developing complications, ${ }^{47}$ timing to specialized care might be important to this group.

The relationship between indirect admission and road distance is likely confounded by nearby trauma centers. Future research will determine the differential distance traveled for indirect admission to understand the role of the spatial relationship among trauma centers, RHSCIR acute facilities, and place of injury in the triage pattern. The spatial relationship should also be examined in terms of driving time, as it is a decision point in pre-hospital triage protocol.

To gain further insight into triage decisions, mode of transportation (ambulance or private vehicle), and timing of injury will be considered. Time of the day could impact driving times, which is especially relevant given the urban nature of the centers being studied, whereas day of the week (weekday vs. weekend) or the year (holiday) could impact surgeon or staff availability. Extending the analysis to beyond $40 \mathrm{~km}$ would be important, as nearly half of the tSCI occurred in the $40-350 \mathrm{~km}$ range. In addition, process mapping of the pre-hospital care phase would capture the practical decision points involved in triage process. Further, all of this information would be critical for identifying appropriate solutions to enhance the triage guideline to support early transport to a SCI center.

\section{Limitations}

Our analysis required location information (FSA), which was collected from patients for whom expanded data were available, and a selection bias was introduced by excluding those without FSA data. However, given that the excluded group shared similar attributes (age and injury mechanism) with those who were injured farther than $40 \mathrm{~km}$ from a RHSCIR acute facility, this exclusion would not be expected to impact our findings, which concerned injuries within $40 \mathrm{~km}$ of a RHSCIR acute facility.

FSA did not provide precise information on injury location, and might affect the accuracy of the road distance measured. ISS was collected through the trauma registry, which primarily included patients with an ISS $>12$. This study was also limited by the small sample size of injuries occurring within $40 \mathrm{~km}$, which prevented stratification by province for adjusting in the multivariable analysis. Finally, provincial triage protocols were not easily accessible, making it difficult to compare practice with the recommendations for each provincial trauma system.

\section{Conclusion}

The geographical pattern of tSCI discovered in this study can inform province-specific resource allocation and triage protocol to optimize the pre-hospital care within a Canadian context. Our findings that $77 \%$ of injuries located in proximity to a RHSCIR acute facility are directly admitted to indicate potential to improve early transport to SCI care. Further, our findings that older age and longer road distance decrease the likelihood of direct admission suggest that lack of recognizing tSCI at the injury scene by paramedics and lack of SCI center designation might contribute to the delayed access to SCI care. This study will inform further research into factors that influence triage and optimal pre-hospital care for individuals with tSCI, and ultimately inform the development of indicators for quality improvement.

\section{Acknowledgments}

The authors thank Tian Shen for analysis support; Tova Plashkes and Barry White for advice; the Rick Hansen Institute RHSCIR Team (Daniel Rogers, Kristen Walden, Jessica Eapen, Jerome Buenaventura, Arlene Aspinall, Cynthia Morin, and Shannon Sproule); and the RHSCIR Network and all of the participating facilities: Vancouver General Hospital, G.F. Strong Rehabilitation Centre, Foothills Medical Centre, Royal Alexandra Hospital, University of Alberta Hospital, Glenrose Rehabilitation Hospital, Royal University Hospital, Saskatoon City Hospital, Winnipeg Health Sciences Centre, St. Michael's Hospital, Sunnybrook Health Sciences Centre, Toronto Western Hospital, Toronto Rehabilitation Institute - Lyndhurst Centre, Hamilton General Hospital, Hamilton Health Sciences - Regional Rehabilitation Centre, University Hospital (London), Victoria Hospital (London), Parkwood Hospital (London), The Ottawa Hospital - Civic Campus, The Ottawa Hospital Rehabilitation Centre, Hôpital de l'Enfant Jésus, Institut de réadaptation en déficience physique de Québec, Hôpital du Sacré-Coeur de Montréal, Centré de réadaptation LucieBruneau, Institut de réadaptation Gingras-Lindsay-de-Montréal, 
QEII Health Sciences Centre, Nova Scotia Rehabilitation Centre, Saint John Regional Hospital, Stan Cassidy Centre for Rehabilitation, St. John's Health Sciences Centre, and Dr. Leonard A. Miller Rehabilitation Centre. This study was supported by financial contributions from the Rick Hansen Institute, the Ontario Neurotrauma Foundation, and the Government of Canada through Health Canada and Western Economic Diversification Canada.

\section{Author Disclosure Statement}

No competing financial interests exist.

\section{References}

1. Tator, C.H., and Fehlings, M.G. (1991). Review of the secondary injury theory of acute spinal cord trauma with emphasis on vascular mechanisms. J. Neurosurg. 75, 15-26.

2. Ahn, H., Singh, J., Nathens, A., MacDonald, R.D., Travers, A., Tallon, J., Fehlings, M.G., and Yee, A. (2011). Pre-hospital care management of a potential spinal cord injured patient: a systematic review of the literature and evidence-based guidelines. J. Neurotrauma 28, 1341-1361.

3. Bourassa-Moreau, É., Mac-Thiong, J.-M., Ehrmann Feldman, D., Thompson, C., and Parent, S. (2013). Complications in acute phase hospitalization of traumatic spinal cord injury: does surgical timing matter? J. Trauma Acute Care Surg. 74, 849-854.

4. Parent, S., Barchi, S., LeBreton, M., Casha, S., and Fehlings, M.G. (2011). The impact of specialized centers of care for spinal cord injury on length of stay, complications, and mortality: a systematic review of the literature. J. Neurotrauma 28, 1363-1370.

5. Theodore, N., Aarabi, B., Dhall, S.S., Gelb, D.E., Hurlbert, R.J., Rozzelle, C.J., Ryken, T.C., Walters, B.C., and Hadley, M.N. (2013). Transportation of patients with acute traumatic cervical spine injuries. Neurosurgery 72, Suppl. 2, 35-39.

6. Maharaj, M.M., Hogan, J.A., Phan, K., and Mobbs, R.J. (2016). The role of specialist units to provide focused care and complication avoidance following traumatic spinal cord injury: a systematic review. Eur. Spine J. 25, 1813-1820.

7. Furlan, J.C., Noonan, V., Cadotte, D.W., and Fehlings, M.G. (2011). Timing of decompressive surgery of spinal cord after traumatic spinal cord injury: an evidence-based examination of pre-clinical and clinical studies. J. Neurotrauma 28, 1371-1399.

8. Grassner, L., Wutte, C., Klein, B., Mach, O., Riesner, S., Panzer, S., Vogel, M., Bühren, V., Strowitzki, M., Vastmans, J., and Maier, D. (2016). Early decompression $(<8$ h) after traumatic cervical spinal cord injury improves functional outcome as assessed by Spinal Cord Independence Measure after one year. J. Neurotrauma 33, 1658-1666.

9. Fehlings, M.G., Cadotte, D.W., and Fehlings, L.N. (2011). A series of systematic reviews on the treatment of acute spinal cord injury: a foundation for best medical practice. J. Neurotrauma 28, 1329-1333.

10. Middleton, P.M., Davies, S.R., Anand, S., Reinten-Reynolds, T., Marial, O., and Middleton, J.W. (2012). The pre-hospital epidemiology and management of spinal cord injuries in New South Wales: 2004-2008. Injury 43, 480-485.

11. Battistuzzo, C.R., Armstrong, A., Clark, J., Worley, L., Sharwood, L., Lin, P., Rooke, G., Skeers, P., Nolan, S., Geragthy, T., Nunn, A., Brown, D.J., Hill, S., Alexander, J., Millard, M., Cox, S.F., Rao, S., Watts, A., Goods, L., Allison, G.T., Laurenson, J., Cameron, P., Mosley, I., Liew, S.M., Geddes, T., Middleton, J., Buchanan, J., Rosenfeld, J. V, Bernard, S., Atresh, S., Patel, A., Schouten, R., Freeman, B.J., Dunlop, S.A., and Batchelor, P.E. (2016). Early decompression following cervical spinal cord injury: examining the process of care from accident scene to surgery. J. Neurotrauma 33, 1161-1169.

12. Wilson, J.R., Voth, J., Singh, A., Middleton, J., Jaglal, S.B., Singh, J.M., Mainprize, T.G., Yee, A., and Fehlings, M.G. (2016). Defining the pathway to definitive care and surgical decompression after traumatic spinal cord injury: results of a Canadian population based cohort study. J. Neurotrauma 33, 963-971.

13. Middleton, J., Sharwood, L.N., Cameron, P., Middleton, P., Harrison, J.E., Brown, D., McClure, R., Smith, K., Muecke, S., and Healy, S. (2014). Right care, right time, right place: improving outcomes for people with spinal cord injury through early access to intervention and improved access to specialised care: study protocol. BMC Health Serv. Res. 14, 600.
14. Thompson, C., Feldman, D.E., and Mac-Thiong, J.-M. (2016). Surgical management of patients following traumatic spinal cord injury: identifying barriers to early surgery in a specialized spinal cord injury center. J. Spinal Cord Med. Apr 8, 1-7.

15. Furlan, J.C., Tung, K., and Fehlings, M.G. (2013). Process benchmarking appraisal of surgical decompression of spinal cord following traumatic cervical spinal cord injury: opportunities to reduce delays in surgical management. J. Neurotrauma 30, 487-491.

16. Acosta, C.D., Kit Delgado, M., Gisondi, M.A., Raghunathan, A., D'Souza, P.A., Gilbert, G., Spain, D.A., Christensen, P., and Wang, N.E. (2010). Characteristics of pediatric trauma transfers to a level I trauma center: implications for developing a regionalized pediatric trauma system in california. Acad. Emerg. Med. 17, 1364-1373.

17. Doumouras, A.G., Haas, B., Gomez, D., de Mestral, C., Boyes, D.M., Morrison, L.J., Craig, A.M., and Nathens, A.B. (2012). The impact of distance on triage to trauma center care in an urban trauma system. Prehosp. Emerg. Care 16, 456-462.

18. Sunnybrook-Osler Centre for Prehospital Care (2009). Toronto Emergency Medical Services Paramedic Guide. The Sunnybrook Centre for Prehospital Medicine, Toronto, Ontario, Canada.

19. Santos, A., Gurling, J., Dvorak, M.F., Noonan, V.K., Fehlings, M.G., Burns, A.S., Lewis, R., Soril, L., Fallah, N., Street, J.T., Bélanger, L., Townson, A., Liang, L., and Atkins, D. (2013). Modeling the patient journey from injury to community reintegration for persons with acute traumatic spinal cord injury in a Canadian centre. PLoS One 8, e72552.

20. Noonan, V.K., Soril, L., Atkins, D., Lewis, R., Santos, A., Fehlings, M.G., Burns, A.S., Singh, A., and Dvorak, M.F. (2012). The application of operations research methodologies to the delivery of care model for traumatic spinal cord injury: the access to care and timing project. J. Neurotrauma 29, 2272-2282.

21. Hameed, S.M., Schuurman, N., Razek, T., Boone, D., Van Heest, R., Taulu, T., Lakha, N., Evans, D.C., Brown, D.R., Kirkpatrick, A.W., Stelfox, H.T., Dyer, D., van Wijngaarden-Stephens, M., Logsetty, S., Nathens, A.B., Charyk-Stewart, T., Rizoli, S., Tremblay, L.N., Brenneman, F., Ahmed, N., Galbraith, E., Parry, N., Girotti, M.J., Pagliarello, G., Tze, N., Khwaja, K., Yanchar, N., Tallon, J.M., Trenholm, J.A.I., Tegart, C., Amram, O., Berube, M., Hameed, U., and Simons, R.K. (2010). Access to trauma systems in Canada. J. Trauma 69, 1350-1361.

22. Noonan, V.K., Chan, E., Santos, A., Soril, L., Lewis, R., Singh, A., Cheng, C.L., O'Connell, C., Truchon, C., Paquet, J., Christie, S., Ethans, K., Tsai, E., Ford, M., Drew, B., Linassi, A.G., Bailey, C.S., Fehlings, M.G., and RHSCIR Network. (2017). Traumatic spinal cord injury care in Canada: A survey of Canadian centres. J. Neurotrauma. [Epub ahead of print. DOI: 10.1089/neu.2016.4928.]

23. Noonan, V.K., Kwon, B.K., Soril, L., Fehlings, M.G., Hurlbert, R.J., Townson, A., Johnson, M., and Dvorak, M.F. (2012). The Rick Hansen Spinal Cord Injury Registry (RHSCIR): a national patientregistry. Spinal Cord 50, 22-27.

24. Dvorak, M.F., Noonan, V.K., Fallah, N., Fisher, C.G., Finkelstein, J., Kwon, B.K., Rivers, C.S., Ahn, H., Paquet, J., Tsai, E.C., Townson, A., Attabib, N., Bailey, C.S., Christie, S.D., Drew, B., Fourney, D.R., Fox, R., Hurlbert, R.J., Johnson, M.G., Linassi, A.G., Parent, S., and Fehlings, M.G. (2014). The influence of time from injury to surgery on motor recovery and length of hospital stay in acute traumatic spinal cord injury: an observational Canadian cohort study. J. Neurotrauma $32,645-654$.

25. Ottenbacher, K.J., and Graham, J.E. (2007). The state-of-the-science: access to postacute care rehabilitation services. A review. Arch. Phys. Med. Rehabil. 88, 1513-1521.

26. Baker, S.P., O'Neill, B., Haddon, W., and Long, W.B. (1974). The injury severity score: a method for describing patients with multiple injuries and evaluating emergency care. J. Trauma 14, 187-196.

27. Waring, W.P., Biering-Sorensen, F., Burns, S., Donovan, W., Graves, D., Jha, A., Jones, L., Kirshblum, S., Marino, R., Mulcahey, M.J., Reeves, R., Scelza, W.M., Schmidt-Read, M., and Stein, A. (2010). 2009 review and revisions of the International Standards for the Neurological Classification of Spinal Cord Injury. J Spinal Cord Med $33,346-352$.

28. Statistics and Research Canada. (2015). Forward Sortation Area - Definition. Innov. Sci. Econ. Dev. Canada. Available at: https://www.ic.gc. ca/eic/site/bsf-osb.nsf/eng/br03396.html. Accessed May 12, 2016.

29. British Columbia Ambulance Serivce (2010). Trauma Destination Decision Guideline. Emergency and Health Services Commission, Vancouver, Canada. 
30. Statistics Canada (2011). Table 051-0001 Estimates of Population, by age group and sex for July 1, Canada, provinces and territories, annual (persons unless otherwise noted), CANSIM (database).

31. Emergency Health Service Branch (2014). Field Trauma Triage and Air Ambulance Utilization Standards. Training Bulletin 113 version 1.0 .

32. Gomez, C.R. (1993). Editorial: Time is brain! J. Stroke Cerebrovasc. Dis. 3, 1-2.

33. Sommer, J.L., and Witkiewicz, P.M. (2004). The therapeutic challenges of dual diagnosis: TBI/SCI. Brain Inj. 18, 1297-1308.

34. Raj, R., Siironen, J., Kivisaari, R., Kuisma, M., Brinck, T., Lappalainen, J., and Skrifvars, M.B. (2013). Factors correlating with delayed trauma center admission following traumatic brain injury. Scand. J. Trauma. Resusc. Emerg. Med. 21, 67.

35. Selvarajah, S., Haider, A.H., Schneider, E.B., Sadowsky, C., Becker, D., and Hammond, E.R. (2015). Traumatic spinal cord injury emergency service triage patterns and the associated emergency department outcomes. J. Neurotrauma 32, 2008-2016.

36. Sharma, S., Gomez, D., de Mestral, C., deMestral, C., Hsiao, M., Rutka, J., and Nathens, A.B. (2014). Emergency access to neurosurgical care for patients with traumatic brain injury. J. Am. Coll. Surg. 218, 51-57.

37. Kuimi, B.L.B., Moore, L., Cissé, B., Gagné, M., Lavoie, A., Bourgeois, G., Lapointe, J., and Jean, S. (2015). Access to a Canadian provincial integrated trauma system: a population-based cohort study. Injury 46, 595-601.

38. Ahn, H., Bailey, C.S., Rivers, C.S., Noonan, V.K., Tsai, E.C., Fourney, D.R., Attabib, N., Kwon, B.K., Christie, S.D., Fehlings, M.G., Finkelstein, J., Hurlbert, R.J., Townson, A., Parent, S., Drew, B., Chen, J., and Dvorak, M.F. (2015). Effect of older age on treatment decisions and outcomes among patients with traumatic spinal cord injury. CMAJ 187, 873-80.

39. Amin, A., Bernard, J., Nadarajah, R., Davies, N., Gow, F., and Tucker, S. (2005). Spinal injuries admitted to a specialist centre over a 5-year period: a study to evaluate delayed admission. Spinal Cord 43, 434 437

40. Bell, N., Simons, R., Hameed, S.M., Schuurman, N., and Wheeler, S. (2012). Does direct transport to provincial burn centres improve out- comes? A spatial epidemiology of severe burn injury in British Columbia, 2001-2006. Can. J. Surg. 55, 110-116.

41. Fallah, N., Noonan, V., Shum, J., Rivers, C., Street, J., Park, S.E., Chan, E., Plashkes, T., and Dvorak, M. (2016). Exploring the possibility of using artificial neural networks to predict mortality after spinal cord injury. Can. J. Surg. 59, S45.

42. Noonan, V., Fallah, N., Bedi, M., Chan, E., Rivers, C., Street, J., Plashkes, T., and Dvorak, M. (2016). Developing a new index to predict mortality after spinal cord injury using machine learning techniques. Can. J. Surg. 59, S45.

43. Casha, S., and Christie, S. (2011). A systematic review of intensive cardiopulmonary management after spinal cord injury. J. Neurotrauma 28, 1479-1495.

44. Anders, J.F., Adelgais, K., Hoyle, J.D., Olsen, C., Jaffe, D.M., and Leonard, J.C. (2014). Comparison of outcomes for children with cervical spine injury based on destination hospital from scene of injury. Acad. Emerg. Med. 21, 55-64.

45. Kwon, B.K., Okon, E., Hillyer, J., Mann, C., Baptiste, D., Weaver, L.C., Fehlings, M.G., and Tetzlaff, W. (2011). A systematic review of non-invasive pharmacologic neuroprotective treatments for acute spinal cord injury. J. Neurotrauma 28, 1545-1588.

46. Krassioukov, A. V, Furlan, J.C., and Fehlings, M.G. (2003). Medical co-morbidities, secondary complications, and mortality in elderly with acute spinal cord injury. J. Neurotrauma 20, 391-399.

47. Street, J.T., Noonan, V.K., Cheung, A., Fisher, C.G., and Dvorak, M.F. (2015). Incidence of acute care adverse events and long-term health-related quality of life in patients with TSCI. Spine J. 15, 923932.

E-mail: ccheng@rickhanseninstitute.org 